\title{
Multistep organic synthesis using flow chemistry
}

\author{
John A. Glaser
}

Published online: 31 March 2013

(c) Springer-Verlag Berlin Heidelberg (outside the USA) 2013

The technology enabling synthetic chemistry within flow systems has been advanced through remarkable synthetic designs using multistep sequences to conduct synthetic schemes leading to products of high complexity. Continuous flow reactors provide a synthetic platform for the combination of several chemical transformations as a single process. This synthetic strategy enables the chemist to organize multiple flow reactors in sequence where the reaction intermediates are not isolated but passed onto the next reactor component of the sequence. Each stream requires flow rate optimization to sustain required residence times and temperature effects for each reactor. Single reactors can be optimized for the reaction conditions conducted within the reactor but the situation becomes more complex when one reactor precedes another in sequence. Conditions are required to support each synthetic stage and a single reactor cannot be optimized by itself. The fine balance of conditions can lead to success or failure of such a flow system. By-products can be removed in-line through the use of phase-bound scavenging reagents. These small flow reactors have been shown to enable the use of unstable intermediates such as acyl azides formed as part of the Curtius rearrangement. The simplicity of the reactor sequence permits consideration of splitting synthetic sequences to permit the desired reaction control. An example of multistep synthesis is shown for the neolignin natural product grossamide. A system of three independent pumps delivering reactants through a series of valves using

\section{J. A. Glaser ( $\square)$}

National Risk Management Research Laboratory, US

Environmental Protection Agency, 26 W King Dr,

Cincinnati, OH 45268, USA

e-mail: Glaser.John@epa.gov in-line monitoring and feedback control was operated under computer control.

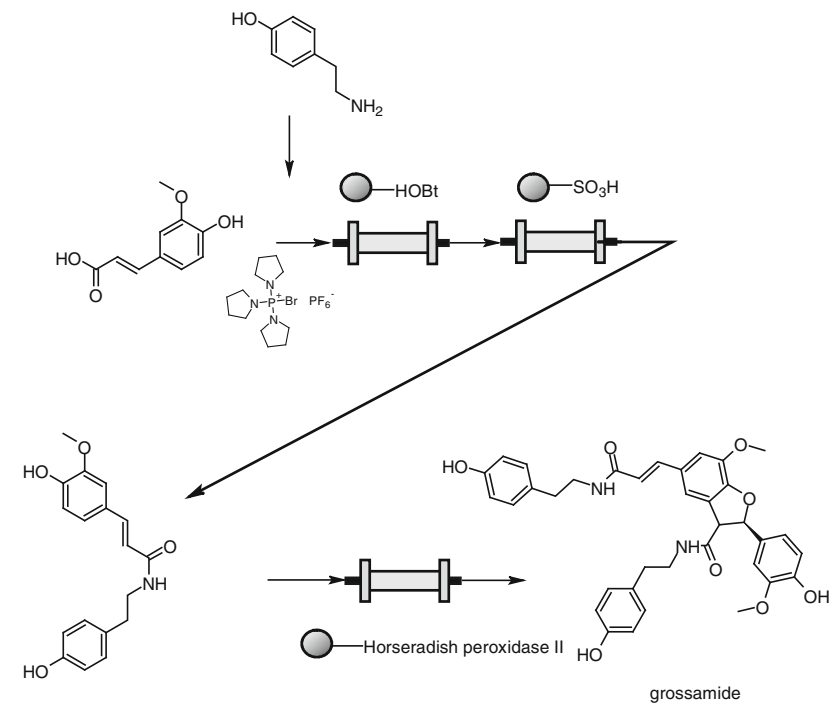

Chem Record 2012, 12, 378-390; Adv Synth Catal 2012, $354,17-57$

\section{Vaccine producing tobacco plants}

Disease prevention through vaccination is a significant achievement that has recently seen major hurdles to accomplishing on-time delivery of the vaccine in necessary quantities. Vaccines have been valued for their prevention of more than 3 million deaths yearly with a positive economic impact estimated to be in excess of $\$ 3$ billion (US). The 2009-2010 swine H1N1 vaccine delivery did not provide sufficient quantities on a timely basis. Fortunately, the flu-based mortality rate was considerably reduced for that season. The 
vaccine for this event was derived from a traditional egg-based manufacturing strategy. New technology development addressing the discovery, assessment, development, and production of vaccines continues to offer the potential to prevent occurrences of past difficulties and enable the discovery and use of new vaccines. Promising technology approaches are plant-based and gene-based vaccines, virus-like particles, novel adjuvants, and delivery systems. The use of tobacco as a host plant has been exploited to produce influenza vaccines. The Australian tobacco plant, Nicotiana benthalamaina, provides the manufacturing platform from which bulk production of vaccines becomes possible. The production sequence begins with the gene synthesis of the appropriate sequence from a targeted virus. Introduction of the genetic material in plants is accomplished through vacuum infiltration. Plants are incubated to effect protein expression and virus-like particle (VLP) formation. The VLPs are extracted from harvested material followed by purification to clinical grade material and then packaged. This process has been evaluated and found to deliver the VLPs in 19 days in contrast to the longer delivery timeframes for alternative technologies. Clearly, management of the yearly flu season has become more problematic for the public health authorities. The consequences of undependable production technology could be catastrophic to the global human population. This new technology is one in a range of options exhibiting attractive features enabling a greater proficiency at combating disease.

Gen Eng Biotech News 2012, 12:6, 8 \& 10

\section{Corporate sustainability reporting advances}

According to the Global Reporting Initiative Annual Report 2011/12, corporate sustainability reporting has become a standard company practice. Published by the Global Reporting Initiative (GRI), a nonprofit organization, the output of a sustainability reporting framework is highlighted in this annual document showing the changes in sustainability reporting within North America and across the globe. The US has contributed to most of the $27 \%$ increase over 2011 numbers. The US reporting for 2011 shows that the number of companies according to GRI standards increased by $44 \%$ over 2010 .

Ninety-five percent of the 250 largest companies of the world report sustainability performance and greater than $80 \%$ of these firms use GRI guidelines. Government guidelines account for some of the change for Sweden, South Africa, France, China, and India. Listing requirements, reporting recommendations, and stock exchange policies influence companies to comply with reporting requirements. The reporting effort has been extended to involve the entire supply chain.
https://www.globalreporting.org/resourcelibrary/GRIAnnual-Report-2011-2012.pdf

\section{Renewable energy goals set by Fortune 100 companies}

A 45-page report, Power Forward: Why the World's Largest Companies are Investing in Renewable Energy, issued by the World Wildlife Fund, CERES, and Calvert Industries, shows that more than half of the US Fortune 100 companies have established greenhouse gas reduction commitments and/or renewable energy commitments. According to interviews with company leaders and public disclosures "clean energy practices are becoming standard procedures," for a group of large and profitable companies. This analysis found that among Fortune's Global 100, twothirds of the companies have made the same commitments. The use of renewable energy is expanding by the world's largest companies "because it makes good sense." Furthermore, the companies find value in a diversified energy supply, hedge fuel cost risk, and energy emissions controls. Renewable energy certificates, power purchase agreements, and on-site direct investments are cited as tools for companies to achieve renewable energy commitments. The report identified the following barriers to renewable energy investment: cost of renewable energy, internal competition for capital, and public policy. A series of recommendations are offered for companies and policy makers which are intended to enhance renewable energy use by companies across the globe.

http://www.ceres.org/resources/reports/power-forward-whythe-world2019s-largest-companies-are-investing-in-rene wable-energy

\section{Caprolactam production environmental impact}

An estimate of the environmental impacts of Chinese caprolactam production was evaluated using the LCA model, IMPACT2002+. Caprolactam is important as the monomer of Nylon 6 which is a component of a wide variety of consumer products; Chinese caprolactam imports provide $60 \%$ of the input needs to Chinese production. The relatively low efficiency and high environmental pollution associated with caprolactam production has attracted the scrutiny of government planners. A caprolactam production of the China Petroleum \& Chemical Corporation was studied as a high-grade petrochemical production example. The process scheme begins with benzene and through a series of interconnected chemical steps leads to caprolactam in commercial quantities as outlined below. 


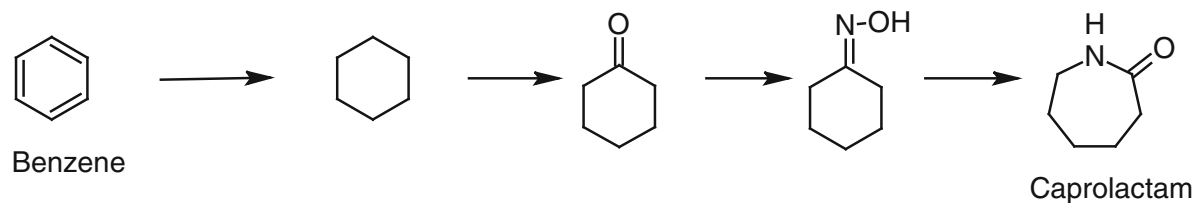

Scheme for caprolactam production

Production and emission data were provided by the production site operator. Life cycle inventory analysis (LCIA) was used to determine the environmental impacts generated by caprolactam production at a Chinese site. The IMPACT2002+ tool was found to be reliable by the authors in the limited context of this investigation. The greatest environmental impact was found with carcinogens, respiratory inorganics, global warming, and non-renewable energy categories. Terrestrial ecotoxicity and non-carcinogenic exhibited a small role with no impact on the other categories considered. As expected, chemical production processes and energy were large contributors to the environmental impact of a caprolactam production site. Surprisingly, caprolactam recovery from recycled nylon filament was recommended for the reduction of potential impact at a caprolactam production site.

J Clean Product 2012, 27, 103-108

\section{Resistance to GM crop traits found with maize pests}

Maize varieties have been genetically engineered to produce insecticidal toxins derived from the bacterium Bacillus thuringiensis (Bt) to control corn pests. Specific traits imparted to maize varieties can control for the western corn rootworm (Diabrotica virgifera virgifera) that significantly lowers the yield. Severe root injury to Cry3Bb1 maize was identified in Iowa, USA beginning in 2009. Field populations of the western corn rootworm, collected and subjected to laboratory bioassays, exhibited survival on Cry3Bb1 maize that was 3 times greater than infield populations unexposed to the western corn rootworm. Of 14 sampled maize fields in Iowa during 2010, root injury was encountered in $50 \%$ of the fields. The fields of the 2010 survey had a history of continuous maize production and 3-7 years cultivation using the Cry3Bb1 hybrid line. No resistance with the Cry34/Cry35Ab1 hybrid lines was encountered. There was no correlation between the two GM strains indicating that cross resistance had not been found. Root injury due to the western corn rootworm was found in fields of the Cry3Bb1 but not with other Bt trait maize types or when the roots were protected by a soil insecticide. Naturally, these results are troubling since rootworm infestation can lead to significant harvest problems which translate into yield reduction. Food and renewable energy demands are based on a dependable supply of the maize crop. Yield reductions only exacerbate market demands. It is desirable that corn rootworm resistance to the $\mathrm{Bt}$ trait resistance can be managed without the loss of any Bt trait.

GM Crops \& Food 2012, 3, 1-10; J Invert Pathol 2012, 1110, 287-293

\section{Energy costs assisted by energy conservation standards}

The American Council for an Energy-Efficient Economy has released a 76-page report, The Efficiency Boom: Cashing in on the Savings from Appliance Standards, which details the effects of new US energy efficiency standards. Mandatory standards are issued by the US Department of Energy. New standards for appliances, industrial equipment, and other products are estimated to save US consumers and businesses $\$ 1.1$ trillion in energy costs by 2035 . The cumulative energy savings is projected to exceed 200 quads or roughly double the US annual energy consumption and reduce $\mathrm{CO}_{2}$ emissions by 470 million tons. The established standards for lighting, appliances, and equipment have been shown to deliver more energy savings than with any other energy consumption sector. The timing of release of the new standards has been slowed by regulatory scrutiny so that the anticipated energy savings will be delayed. This analysis shows that consumers and business will suffer a loss of $\$ 300$ million in savings with the emission of another 4.4 million metric tons to the atmosphere from this delay.

http://aceee.org/research-report/a123; http://www.appliancestandards.org/sites/default/files/The_Cost_of_Overdue_Ene rgy_Efficiency_Standard_Jan_2013_0.pdf

\section{Biologically based plastics}

The worldwide production of plastics is expected to exceed 300 million tons during the period of 2010-2012. Derived largely from petroleum, plastics production/manufacture will emit hundreds of millions of tons of $\mathrm{CO}_{2}$ with other emissions having human health risks. Short-term plastic consumption accounts for $40 \%$ of the total consumption. 
The wastes associated with petrochemical plastic production have been found to be harmful to terrestrial and sea animals. It is incumbent on society to undertake a technical evaluation of plastic production from bio-based sustainable chemicals for their business needs. The Guide to Safer Chemicals version 1 lists specific tools to provide chemical hazard information and specific information for selected chemicals identified by government agencies for additional<smiles>OCCCO</smiles>

1,3-Propanediol

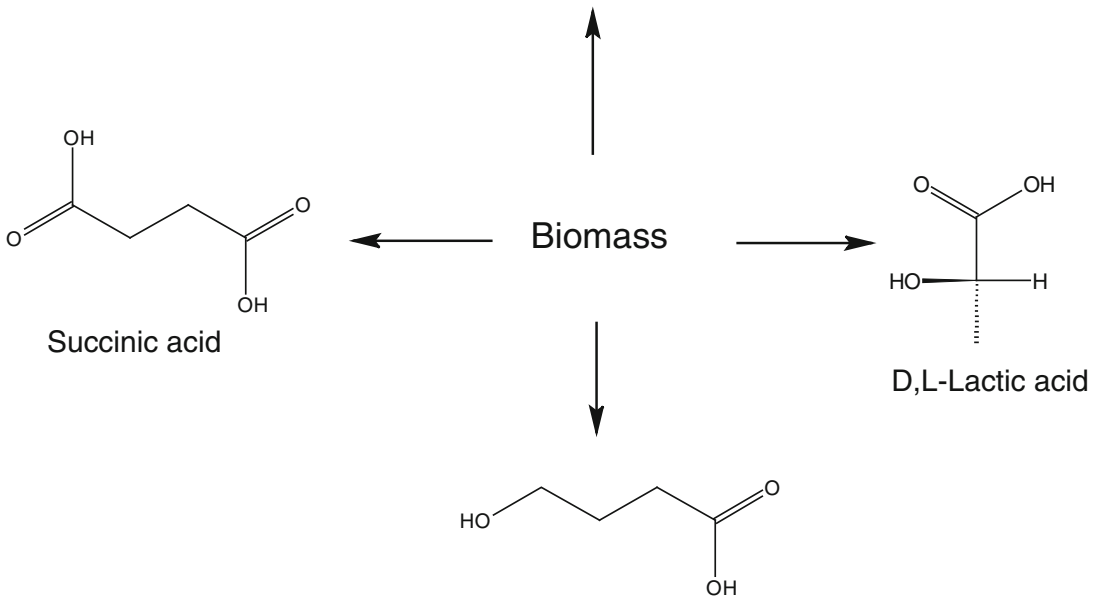

3-Hydroxypropionic acid

resources. Examples of the types and diversity of monomers available from raw materials (biomass) of the environment are depicted in the following drawing.

Biological sources of monomers include starch, cellulose, proteins, sugars, fatty acids, and other complex materials. Microbiological consumption of these renewable materials can be selectively guided to form organic monomers. Organism selection and molecular manipulation of the microbe have been shown to offer increasingly attractive and economic means for monomer production. In the case of the four monomers depicted in the figure, biobased plastics such as polyhydroxyalkanoates, polylactic acid, poly(butylene succinate), and poly(trimethylene terephthalate) are targets of current process development. If optically active lactic acid is polymerized, the resulting polymer has very interesting properties such as high degree of crystallinity and higher glass transition temperature. These properties can offer more opportunities to the polymer processor to formulate polymer blends having greater consumer utility and appeal.

Chem Rev 2012 112.2082-2099

\section{Safe chemicals guide}

Formed from a coalition and a committee, BizNGO, has released a 60-page guide to help companies select safer concern. A web-based tool, Clean Production Action's GreenScreen, enables companies to evaluate 18 potential problems related to chemical selection such as carcinogenicity, human reproduction-related issues, or possibility of ecosystem damage. The guide is advanced partially to provide information relating to the safety of chemicals and address the concern of consumers. The strategies and resources offered to companies through this publication are expected to assist the implementation of safer chemical use principles.

http://www.bizngo.org/guide.php

\section{Rising global temperature consequences}

The World Bank, together with the Potsdam Institute for Climate Research and Climate Analytics, has released an 84-page report, Turn Down the Heat: Why a $4{ }^{\circ} \mathrm{C}$ Warmer World Must be Avoided, that explores in a quantitative manner the potential impacts of $4{ }^{\circ} \mathrm{C}$ or $7.2{ }^{\circ} \mathrm{F}$ warming on developing countries. The report is organized into seven sections. After the introduction, observed climate changes and inputs are vetted. The next section addresses $21 \mathrm{st}$ century projection for the changes and impacts. This is followed by sea-level rise projections evaluated across the globe. Next, changes in extreme temperatures are scrutinized both spatially and temporarily. Impacts within and 
across the sectors of agriculture, water resources, ecosystems and biodiversity, and human health are shown for the current level of research and complications. These new analyses of regional sea-level rise and extreme heat wave increases require cross sector risk assessment with attention given to system interaction and non-linearity. A complement of 39 figures offers a rough estimate of the analytical depth of the report.

The global impact of a $4{ }^{\circ} \mathrm{C}$ increase could lead to extreme heat waves during the summer months in many regions including the US, sea-level rise of $1.6-3.2 \mathrm{ft}$ by 2100 , enhanced water scarcity problems, population displacement at a large scale, weather changes (tropical cyclone intensity increase), and irreversible biodiversity losses. The report asserts that these conditions can be significantly reduced by limiting the global temperature rise to $2{ }^{\circ} \mathrm{C}$, the agreed upon international goal.

Price Waterhouse Cooper released a complementary report, Too Late for Two Degrees? Low Carbon Economy Index 2012, in which companies are warned to prepare for a $6{ }^{\circ} \mathrm{C}$ rise in the global temperature by the turn of the century. The rates of carbon emission reduction (decarbonization) per unit of gross domestic product in major economies or carbon intensity have passed critical thresholds. At current levels of carbon emission, abatement shows a gap of $12 \mathrm{Gt} \mathrm{CO}_{2}$ by $202030 \mathrm{Gt} \mathrm{CO}_{2}$ by 2030 and $70 \mathrm{Gt} \mathrm{CO}_{2}$ by 2050 when compared with a targeted $2{ }^{\circ} \mathrm{C}$ rise. A separate report, Risk Ready: New Approaches to Environmental and Social Change, emphasizes that climate models project global temperatures to rise more sharply to levels beyond the accepted norm for keeping Earth's systems within stable conditions. Recent weather-related events are offered as signature events of the perilous conditions for the global community to endure.

Companies are advised to create buffers or margins designed to absorb shocks accompanying climate change such as resource scarcity. Adaptive measures to population growth, water use, energy requirements, and dwindling resources are recommended to companies as means to increase corporate resilience.

http://climatechange.worldbank.org/sites/default/files/Turn_ Down_the_heat_Why_a_4_degree_centrigrade_warmer_ world_must_be_avoided.pdf; http://pwc.to/VN6LAh; http:// pwc.to/PoVBn1

\section{Permafrost changes}

A 30-page UNEP report Policy Implications of Warming Permafrost, released in 2012, underscores the growing concern for permafrost thawing in colder climates due to anticipated increases in the global temperature of $3^{\circ}$. This temperature rise would translate to a $6^{\circ}$ increase in the Arctic. Permafrost is found in about $24 \%$ of the land surface of the northern hemisphere. Vast amounts of methane and carbon dioxide are stored under the earth's surface in the permafrost layers. The report shows that monitoring efforts have already found evidence to suggest large-scale thawing of the permafrost, and carbon emissions as the trapped methane and carbon dioxide are released is known as the permafrost carbon feedback. The permafrost layers of the Earth can run to 700-meter depths and store the equivalent of $1,700 \mathrm{Gt}$ of $\mathrm{CO}_{2}$, which is almost twice the current atmospheric concentration of $\mathrm{CO}_{2}$. Additional emissions in the range of $43-135$ Gt are anticipated by the turn of the century. An estimate of $246-415$ Gt in emissions could occur by 2200 . As the global temperature rises, more of the permafrost thaws and releases the contained GHGs. Should the globe experience widespread loss of permafrost significant environmental impacts such as local hydrology changes, an increase in fire frequency, loss of wetlands and lakes, and damage to critical infrastructure as roads and buildings could be anticipated. A global temperature increase of 3 or $6{ }^{\circ} \mathrm{C}$ in the Arctic could result in the loss of 30-85\% of the global permafrost layer. With the release of the entrapped GHG components, the global temperature is expected to rise higher.

http://www.unep.org/pdf/permafrost.pdf

\section{Green chemistry panorama}

The Royal Society of Chemistry has published a single issue of Chemical Society Reviews of 190 pages with 11 articles and focused on different aspects of the emerging effort to provide chemical products and processes designed to reduce or eliminate the use or generation of hazardous substances. The articles are subdivided into two groups of tutorial and critical reviews. The tutorial reviews are devoted to organic synthesis in water, supercritical carbon dioxide as a reaction medium, reaction design efficiency, process research, cobalt catalyzed coupling of carbon dioxide and epoxides, and evaluating greenness. The critical reviews address renewable surfactant synthesis, cellulose treatment with ionic liquids, selected chemicals from biomass, alternative energy sources for organic synthesis, and development of an organic toolbox for biocatalysis integration. The themed issue provides the opportunity for selected researchers to provide background and achievement current to the emerging practice of green chemistry. One example of successful green chemistry applied at the process level is the synthesis of pregabalin $\left(\right.$ Lyrica $\left.^{\circledR}\right)$. The 
classic route to pregabalin was severely hampered by the resolution of a racemic mixture. The yield of pregabalin was about $30 \%$ with the remaining $70 \%$ of the material mass lost as waste during this step leading to $86 \mathrm{~kg}$ of waste generated for every kilogram of pregabalin produced. To improve the process from both material and cost considerations, the development of a biocatalysis route was investigated and found to be highly desirable. The selected enzyme permitted the entire process to be conducted in water. Further improvements were made and a synthetic route involving recycle was evaluated and commercialized. An analysis of the E factor was conducted for the three synthetic routes. The results are in the accompanying figure. A remarkable tenfold reduction of the $\mathrm{E}$ factor was found when the 1st (classic) route was compared with the last. This remarkable effort shows how synthetic routes can be manipulated to enhance the reduction of waste and cost of manufacture.<smiles>CCOC(=O)C(C#N)C(CC(C)C)C(=O)OCC</smiles>

Cyanodiester

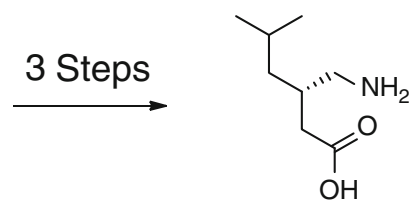

Pregabalin

$\begin{array}{cc}\text { Process } & E \text { factor } \\ \text { Classic } & 86 \\ \text { Biocatalysis } & 17 \\ \text { Recycle } & 8\end{array}$

\section{Sustainability assessment uncertainty}

The assessment of technologies, processes, products, and policies requires comprehensive approaches that enable the judging of these developments in robust and reliable ways that meet the needs of the present without compromising similar evaluations related to future evaluative needs. The EC Joint Research Centre has released a technical report, Dealing with uncertainty in sustainability assessment, that evaluates field-specific simulation models through the application of sensitivity analysis techniques. For a framework of sustainability assessment it is necessary to identify the major sources of uncertainty. The assessment of a conceptualized sustainable development must begin with boundary definitions, an understanding of assessment tools and their vulnerability to subjectivity, and the recognition of a model's inability to properly mimic the real world. A conceptual framework becomes necessary to guide the analysis to more completely understand the limits of our comprehension of the real world. The main sources of uncertainty are considered initially to assist the formulation of a conceptual framework to assist the sustainability assessment. Widely adopted techniques for sensitivity and uncertainty analysis are scrutinized and applied to a test scenario. The importance of understandable, valid, and pertinent data derived from sustainability analysis for a given scenario becomes very important to the interested public, and decision makers.

http://publications.jrc.ec.europa.eu/repository/handle/1111 $11111 / 26231$

\section{Process control in process intensification}

Challenges presented to the process industry are diverse and emphasize the development of a sustainable industry that addresses environmental responsibility, enhanced energy use efficiency, and the incorporation of renewable energy use. Considerations of waste production, GHG emissions, and other contaminated media regulations are leading to stringent requirements for process operation. Process intensification in such forms as reactive distillation, simulated moving-bed reactors, and micro-scale reactor systems has been implemented in different industrial applications. Process control can significantly enhance the flexibility, economics, and environmental footprint of these emerging processes. There are significant challenges to the control of process intensification. The authors offer a schema of six layers to direct the development of control and optimization to the state of art for continuous manufacturing. The one through three layers focus on control and the four through six layers optimize the process. A new concept of process synthesis is presented incorporating process intensification and actuation involvement. Dynamic optimization leads to the integration of process operation, design, and control. The approach offers optimal operation and more efficient control of complex systems utilizing process intensification methodology.

\section{Chem Eng Proc Process Inten 2012, 1-15}

\section{Polymer use in sustainable engineering}

An entire issue of the International Journal of Sustainable Engineering focuses on the use of polymers in sustainable engineering. The eight articles cover biobased lubricant blends, thermo-mechanical processing of polymers, cellulosic materials from supercritical $\mathrm{CO}_{2}$ processing, concrete with recycled rubber, carbon 
footprint analysis for recycled e-waste, and green supply chain for plastic films. These different approaches offer significant information and analysis to give direction to new research efforts in the role of polymers for sustainable engineering.

Internat J Sus Engin 2012, 5(1), 1-83

\section{New journal}

Energy Technology is a new journal addition designed to address the multifaceted needs of society relative to applied energy research. Energy process engineering is the focus of the new journal from new concepts, design, to production. As a new voice in the energy area, the journal strives to address the four characteristics of energy research: generation, storage, conversion, and distribution. Complementary online access for 2013 is available.

http://onlinelibrary.wiley.com/journal/10.1002/\%28ISSN\% $292194-4296$ ? dmmsmid $=68207 \& \mathrm{dmmspid}=4763890 \&$ dmmsuid $=1810311$ 